\title{
Die Altersversorgung von Beamten in Tschechien
}

\author{
Martin Štefko
}

\section{Einleitung}

II. Alterssicherungssysteme für Beschäftigte im öffentlichen Dienst

1. Das allgemeine Rentenversicherungssystem

2. Besondere Regelungen für Staatsbedienstete

III. Bewertung 


\section{Einleitung}

Das tschechische Rechtssystem kennt den Status des „Beamten der Tschechischen Republik“ und des „Beamten der Städte und Regionen“.1

Das Arbeitsgesetzbuch normiert als besondere Pflichten des Beamten die Loyalitätspflicht ${ }^{2}$, die Pflicht zur Amtsverschwiegenheit und die Pflicht zur unparteilichen Dienstausübung. Zugleich enthält das Arbeitsgesetzbuch Beschränkungen für zusätzliche Einkünfte der Staatsbediensteten aus einer Nebenbeschäftigung.

In Anbetracht der besonderen Pflichten hat der Gesetzgeber besondere Regelungen für Beamte in verschiedenen Gesetzen geschaffen.

Das Gesetz über Berufssoldaten regelt die Rechtsverhältnisse der Berufssoldaten. ${ }^{3}$

Ein weiteres Spezialgesetz ist das Gesetz über Beamte in territorialen Selbstverwaltungseinheiten. ${ }^{4}$ Dieses Gesetz regelt die Besonderheiten des Arbeitsverhältnisses von Beamten in territorialen Selbstverwaltungseinheiten, insbesondere die Begründung des Beamtenverhältnisses einschließlich des Auswahlverfahrens im Rahmen der Stellenbesetzung (z.B. die besonderen Anforderungen, die ein Bewerber erfüllen muß), die Dauer des Beamtenverhältnisses, die Voraussetzungen für die Versetzung von Beamten, die Amtsenthebung und die Leistungsbereitschaft. Die Vorschriften des Arbeitsgesetzbuches finden auf die arbeitsrechtlichen Verhältnisse der Beamten in territorialen Selbstverwaltungseinheiten Anwendung, sofern das Gesetz Nr. 312/2002 Sb. keine anderweitigen Regelungen getroffen hat. Beispielsweise sieht das Gesetz Nr. 312/2002 Sb. eine höhere Abfindungszahlung bei Beendigung des Dienstverhältnisses vor.

Mit dem Gesetz über das Dienstverhältnis der Mitglieder von bewaffnen Einheiten 5 werden die Rechtsbeziehungen von natürlichen Personen, die in einer bewaffneten Einheit ihren Dienst leisten, geregelt. Hierzu gehören Bedienstete der Polizei der Tschechischen Republik, der Feuerwehr der Tschechischen Republik, der Tschechischen Zollverwaltung, des Tschechischen Gefängnisdienstes, des Sicherheitsinformationsdienstes

1 Vgl. § 2 des Gesetzes Nr. 218/2002 Sb. o službě státních zaměstnanců ve správních úřadech a o odměňování těchto zaměstnanců a ostatních zaměstnanců ve správních úřadech, služební zákon (über Staatsangestellte in Verwaltungsbehörden und über die Entlohnung dieser Angestellten sowie anderer Angestellter in Verwaltungsbehörden - Dienstgesetz) und § 2 Abs. 4 Gesetz Nr. 312/2002 Sb. über Beamte in territorialen Selbstverwaltungseinheiten idF des Gesetzes 264/2006.

Die Beamten der Städte und Regionen sind eigentlich Arbeitnehmer und das Gesetz Nr. 312/2002 Sb. regelt nur geringere Abweichungen vom Arbeitsgesetzbuch.

2 Beispielsweise das Verbot der Entgegennahme von Geschenken oder anderen Vorteilen.

3 Gesetz Nr. 221/1999 o vojácích z povolani (Gesetz über Berufsoldaten) vom 14.9.1999 idF des Gesetzes $272 / 2009$.

4 Gesetz Nr. 312/2002 Sb. o úřednících územních samosprávných celků a o změně některých zákonů (Gesetz über Beamte in territorialen Selbstverwaltungseinheiten) vom 13.6.2002 idF des Gesetzes 264/2006.

5 Gesetz Nr. 361/2003 Sb. o služebním poměru př́slušníků bezpečnostních sborů (über das Dienstverhältnis der Mitglieder von bewaffnen Einheiten) vom 23.9.2003 idF des Gesetzes 306/2008. 
sowie der Behörde für Auslandsbeziehungen und Informationen. Das Gesetz enthält Regelungen zu ihrer Entlohnung, zu Verhandlungen, die im Rahmen eines Dienstverhältnisses geführt werden, sowie organisatorische Angelegenheiten des Dienstes selbst. Im Rahmen des Auswahlverfahrens ist vorgesehen, daß der Bewerber bei der Vorbereitung auf den Dienst bzw. während der Ausbildung einen befristeten Dienstvertrag erhält. Da das Gesetz Nr. 361/2003 Sb. die arbeitsrechtlichen Beziehungen von Mitgliedern der bewaffnen Einheiten umfassend regelt, bleibt für die Anwendung des Arbeitsgesetzbuches wenig Raum.

Für den Staatsdienst, der nicht an der Waffe erfolgt, ist das Dienstgesetz 6 ausgearbeitet worden. Der Gesetzeszweck besteht darin, die rechtlichen Verhältnisse der Angestellten, die in Verwaltungsbehörden öffentliche Dienste leisten, welche die Tschechische Republik der Allgemeinheit anbietet, zu regeln. Die Regulierung der

- organisatorischen Angelegenheiten des Staatsdienstes,

- Vorbereitung natürlicher Personen auf einen solchen Dienst,

- Dienstbeziehungen der Staatsangestellten in Verwaltungsbehörden,

- Entlohnung dieser Angestellten, sofern durch eine spezielle rechtliche Regelung nicht anders geregelt,

- Grundlagen der Dienstausübung,

- Entlohnung anderer, sich bei Staatsbehörden in einem Arbeitsverhältnis befindlichen Angestellten und

- organisatorischen Angelegenheiten, welche die Beschäftigung dieser Angestellten betreffen,

ist, obwohl es erforderlich wäre, nicht erfolgt. Das Dienstgesetz ist bislang nicht in Kraft getreten.

Der Termin für das Inkrafttreten des Dienstgesetzes wurde inzwischen fünfmal verschoben. ${ }^{7}$ Den Hauptgrund für die wiederholte Verschiebung kann man in der Kostspieligkeit des neuen Systems sehen. Das Dienstgesetz hat aber auch das Vertrauen der Subjekte, auf die es angewendet werden sollte, verloren. Während der Vorbereitungsarbeiten am Dienstgesetzentwurf wurden wegen der Kosten nicht nur zahlreiche Rechte und Sozialvorteile für Beamte gestrichen, sondern auch der Anwendungsbereich des Gesetzes bedeutend reduziert. Das Dienstgesetz setzt sich aus zwölf Teilen zusammen: Das Beamtenverhältnis, Rechte und Pflichten der Beamten, die Disziplin und die Arbeitsbedingungen sind in den Teilen drei bis elf geregelt. Für diesen Bericht ist der vierte Teil über Sozialleistungen von Bedeutung. Gemäß $§ 110$ des Dienstgesetzes haben Beamte Anspruch auf zwei wichtige Leistungen: Entgeltfortzahlung im Krankheitsfall und Aus-

6 Gesetz Nr. 218/2002 Sb. o službě státních zaměstnanců ve správních úřadech a o odměňování těchto zaměstnanců a ostatních zaměstnanců ve správních úřadech, služební zákon (über Staatsangestellte in Verwaltungsbehörden und über die Entlohnung dieser Angestellten sowie anderer Angestellter in Verwaltungsbehörden - Dienstgesetz) vom 26.4.2002 idF des Gesetzes 41/2009.

7 Die schrittweise festgesetzten Termine, zu denen das Dienstgesetz mit allen seinen Bestimmungen in Kraft treten sollte, waren 2004, 2005, 2007, 2009 und 2012. 
dienzulage. Die erste Leistung wurde durch die Dienstgesetznovellen ausgehöhlt. Deshalb gibt es zurzeit keinen wesentlichen Unterschied zwischen der Regelung im Dienstgesetz und der im Arbeitsgesetzbuch. Der Rentenzuschuß als Ausdienzulage gebührt den ehemaligen Beamten und ihren Hinterbliebenen. Der ehemalige Beamte kann diese Leistung unter der Bedingung beanspruchen, das der Dienst im Beamtenverhältnis auf unbegrenzte Dauer mindestens fünf Jahre im Zeitraum der letzten 20 Jahre vor dem Tag, an dem die Altersrente oder Invalidenrente zugesprochen wurde, gedauert hat. Die Höhe der Ausdienzulage wird nach ähnlichen Prinzipien wie die allgemeine Rente berechnet. Für jedes zurückgelegte Dienstjahr ist $1 \%$ vom reduzierten Monatsgehalt anzusetzen, das der Beamte durchschnittlich in den letzten fünf Jahren vor Rentengewährung erzielt hat. Die Maximalhöhe der Ausdienzulage beträgt $20 \%$.

Die Arbeitsverhältnisse der meisten Staatsangestellten richten sich auch weiterhin nach den allgemeinen Regeln des Arbeitsrechts.

\section{Alterssicherungssysteme für Beschäftigte im öffentlichen Dienst}

Die Alterssicherung aller erwerbstätigen Personen in der Tschechischen Republik erfolgt über das allgemeine System der (gesetzlichen) Rentenversicherung. Für Beamte ist als zusätzliche Leistung die Gewährung von Zulagen vorgesehen, die nach Ablauf einer bestimmten Dienstzeit ausgezahlt werden. Zudem sind für die Alterssicherung der Beamten verschiedene Versicherungsträger, nämlich das Verteidigungsministerium, das Innenministerium und das Justizministerium zuständig.

\section{Das allgemeine Rentenversicherungssystem}

Das tschechische Rentenversicherungssystem besteht derzeit aus zwei Säulen, nämlich dem einheitlichen System der obligatorischen Rentenversicherung ${ }^{8}$ sowie dem System der Rentenzusatzversicherung ${ }^{9}$, das staatlich bezuschußt wird.

Die obligatorische Rentenversicherung setzt sich aus einem obligatorischen leistungsdefinierten PAYG-Pfeiler ${ }^{10}$ zusammen, der bei der Berechnung der Versicherungsleistungen zugrunde gelegt wird. Ferner ist ein fakultativer beitragsdefinierter Pfeiler vorgesehen.

8 Vgl. $§ 2$ und 5 des Gesetzes Nr. 155/1995 o důchodovém pojištění (über Rentenversicherung) vom 30.6.1995.

9 Vgl. $\S 1$ und 2 des Gesetzes Nr. 42/1994 o penzijním připojištění se státním př́spěvkem a o změnách některých zákonů souvisejících $\mathrm{s}$ jeho zavedením (über Rentenzusatzversicherung mit staatlichem Zuschuß) vom 16.2.1994.

10 PAYG = Pay As You Go.

Renten werden durchlaufend aus angesammelten Versicherungsbeiträgen gezahlt. 
Es gibt folgende Rentenarten:

- Altersrente

- Volle Invalidenrente

- Teilinvalidenrente

- Witwen- bzw. Witwerrente

- Waisenrente

Die Höhe der Rente wird aus einem Grundbetrag und einer prozentualen Bemessung berechnet. Als Grundbetrag wird vom Gesetzgeber ein fester Betrag vorgegeben, ${ }^{11}$ während die prozentuale Bemessung auf Grundlage der persönlichen Bemessungsgrundlage und der Anzahl der Versicherungsjahre berechnet wird. Bei Hinterbliebenenrenten wird der Betrag mittels Applizierung der prozentualen Berechnung auf die Rente des Verstorbenen ermittelt. Sofern es sich um Invalidenrenten handelt, kann die Rentenhöhe neben den Regeln des beitragsdefinierten Rentenplans auch so festgelegt werden, daß der Rentenfonds bei Vorliegen bestimmter Anspruchsvoraussetzungen auch ihre vereinbarte Höhe garantiert, wobei die Höhe evtl. auch anderweitig festgelegt werden kann.

Die obligatorische Rentenversicherung wird aus dem Versicherungsgeld (Beiträge der Arbeitnehmer und Arbeitgeber), das in den Staatshaushalt abgeführt wird, im Umlageverfahren finanziert. Innerhalb des Staatshaushaltes gibt es ein spezielles Konto für die Rentenversicherung. Träger der Rentenversicherung ist die Tschechische Sozialversicherungsanstalt.

Die Rentenzusatzversicherung wird neben den Beiträgen der Versicherten durch staatliche Zuschüsse finanziert. Zwar können auch andere Personen, wie der Arbeitgeber, in die Rentenzusatzversicherung einzahlen. Dadurch werden jedoch keine besonderen Rechte begründet. Die Rentenzusatzversicherung wird staatlich gefördert. Der maximale staatliche Zuschuß beträgt $150 \mathrm{CZK}^{12}$, wobei Versicherte diesen Zuschuß nur erhalten, wenn sie monatlich $500 \mathrm{CZK}$ einzahlen. Zahlen die Versicherten jährlich mehr als $6000 \mathrm{CZK}$ ein, so ist der Teilbetrag, um den ihr Beitrag die Grenze von 6000 CZK übersteigt, von der Steuer absetzbar. Der von der steuerlichen Berechnungsgrundlage diesbezüglich maximal absetzbare Betrag beläuft sich auf $12.000 \mathrm{CZK}^{13}$ jährlich. Der Arbeitgeberbeitrag ist zwar für die Versicherten als Einnahme zu betrachten. Dieser unterliegt aber nicht der Einkommensteuer. Auch werden keine Beiträge für das Gesundheits- und Sozialversicherungssystem abgezogen. Versicherungsträger ist der Rentenfonds. Die Leistungen aus der Rentenzusatzversicherung umfassen

- Rente (Alters-, Invaliden- oder Ausdienrente),

- einmalige Abfindung und

- Apanage.

11 Zurzeit ca. $85,0980 €$ bei einem Kurs von 25,5 Kronen für $1 €$.

12 Ca. $6 €$ bei einem Kurs von 25.5 Kronen für $1 €$.

13 Ca. $470 €$. 
Daneben besteht die Möglichkeit für die Versicherten, eine private Rentenzusatzversicherung bei einer privaten Versicherungsgesellschaft zu begründen.

Beamte sind als Angestellte in der gesetzlichen Rentenversicherung pflichtversichert. Eine Ausnahme besteht für Beamte bzw. sonstige Angestellte der Europäischen Gesellschaften und ihrer Institutionen. Das Gesetz über die Rentenversicherung räumt diesem Personenkreis die Möglichkeit ein, aus dem nationalen System der Rentenversicherung auszutreten. Auch werden Beamte vom Versichertenkreis der Rentenzusatzversicherung erfasst. Mit dem Inkrafttreten des Dienstgesetzes wird die Möglichkeit des Staates, die Altersabsicherung der Beamten durch Zuschüsse in die Rentenzusatzversicherung zu unterstützen, aufgehoben. ${ }^{14}$

\section{Besondere Regelungen für Staatsbedienstete}

Die Rentenversicherung der Beamten wird von anderen Trägern als dem der allgemeinen Rentenversicherung verwaltet:

- Bei Berufssoldaten und Soldaten, die für den Zeitraum des ausgeübten Dienstes materiell wie Berufssoldaten abgesichert sind, ist das Verteidigungsministerium Versicherungsträger.

- Bei Bediensteten der Polizei und der Feuerwehr der Tschechischen Republik, des Sicherheitsinformationsdienstes sowie der Behörde für Auslandsbeziehungen und Informationen ist das Innenministerium Versicherungsträger.

- Bei Bediensteten des Tschechischen Gefängnisdienstes ist das Justizministerium Versicherungsträger.

Bestimmte Gruppen von Beamten können neben den Leistungen aus dem allgemeinen Rentenversicherungssystem verschiedene Arten von Ausdienzulagen aus dem jeweiligen Sondersystem beanspruchen, welche nach Ablauf einer bestimmten Dienstzeit jeweils für eine bestimmte Dauer und in bestimmter Höhe ausgezahlt werden.

Diese besondere Leistung beziehen jedoch nicht die Staatsbediensteten in territorialen Selbstverwaltungseinheiten.

Die Ausdienzulagen werden in $\S \S 157-163$ des Gesetzes über das Dienstverhältnis der Mitglieder von bewaffnen Einheiten und $\S \S 132-137$ des Gesetzes über Berufssoldaten geregelt.

14 Vgl. § 27 Abs. 5 des Gesetzes Nr. 42/1994.

Es gibt einen einheitlichen allgemeinen staatlichen Zuschuß. Die Beamten haben zurzeit keine Vorteile. Das Verbot, die Zuschüsse in die Rentenzusatzversicherung zu entrichten, das im Dienstgesetz enthalten ist, verpflichtet nur den tschechischen Staat. Der Gesetzgeber wollte auf dieser Art die Konkurrenz zwischen Ausdienzulage und staatlichem Zuschuß in die Rentenzusatzversicherung als eine doppelte Vergünstigung der Beamten ausschließen. Ein Beamter kann natürlich auch nach dem Inkrafttreten des Dienstgesetzes eine Rentenzusatzversicherung abschließen, nur der Staat sollte nach dem Dienstgesetz zu einer solchen privaten Rentenzusatzversicherung beitragen. 
Die Ausdienzulagen bei Soldaten und Mitgliedern der bewaffnen Einheiten sind wie folgt ausgestaltet: Ein Berufssoldat hat nach mindestens 15 abgeleisteten Dienstjahren Anspruch auf eine monatliche Ausdienzulage auf dem Basisniveau von $5 \%$ des durchschnittlichen Bruttomonatsgehaltes (dBMG). Mit jedem weiteren abgeleisteten Dienstjahr erhöht sich die Zulage, bis nach 20 Dienstjahren $36 \%$ des dBMG erreicht werden. Die maximale Höhe der Ausdienzulage wird durch das Gesetz Nr. 221/1999 Sb. geregelt und beläuft sich auf $55 \%$ de dBMG. Hat ein Soldat seinen Dienst als aktiver Militärpilot geleistet oder handelte es sich um einen Dienst besonderer Art, war die Ausübung des Dienstes besonders gefährlich oder wurde der Dienst im Ausland unter besonders gefährlichen Bedingungen geleistet, dann beläuft sich die Maximalhöhe der Zulage auf $60 \%$ des dBMG.

Ein Anspruch auf die monatliche Ausdienzulage besteht nach Ablauf von 15 Dienstjahren jedoch nicht, wenn das Dienstverhältnis durch Entlassung beendet wurde.

Die Basishöhe der Ausdienzulage für ein Mitglied einer bewaffneten Einheit beträgt nach 15 abgeleisteten Dienstjahren $20 \%$ des monatlichen Diensteinkommens und erhöht sich nach dem 16. sowie jedem weiteren abgeschlossenen Dienstjahr um weitere $3 \%$ des monatlichen Diensteinkommens. Ab dem 21. und jedem weiteren abgeschlossenen Dienstjahr erhöht sich die Ausdienzulage um je $2 \%$ des monatlichen Diensteinkommens, später nach jedem weiteren abgeschlossenen Dienstjahr um jeweils $1 \%$. Die Maximalhöhe des Ausdienzuschusses beläuft sich in diesem Fall auf $50 \%$ des monatlichen Diensteinkommens.

Das allgemeine Rentenversicherungssystem berücksichtigt die Ausdienzuschüsse bei der Berechnung der Höhe der Altersrente, wie sich am Beispiel der Soldaten sowie der Bediensteten in bewaffneten Einheiten demonstrieren läßt: Bei gleichzeitigem Entstehen eines Anspruches auf die Ausdienzulage und dem Anspruch auf Altersrente, der vollen Invalidenrente oder der Teilinvalidenrente, wird die Ausdienzulage nur dann ausgezahlt, wenn sie betragsmäßig höher ist als eine der aufgeführten Rentenarten, wobei jeweils die Differenz zwischen der Ausdienzulage und der jeweiligen Rente ausgezahlt wird. Bei der Berechnung des Differenzbetrages zwischen der Ausdienzulage und der Rente wird als Referenzdatum der Tag genommen, an dem der Anspruch auf die Altersrente, die volle Invalidenrente oder die Teilinvalidenrente entstand, oder aber der Tag der Erhöhung der Altersrente oder der Anpassung der Teilinvalidenrente, die auf Grund einer Erwerbstätigkeit des Versicherten erfolgte. Anzumerken ist, daß der Versicherte höchstens zwei Jahre nach der Entstehung des Anspruches auf Altersrente einen Zusatzverdienst erzielen darf. Zudem muß der Unterschiedsbetrag innerhalb von zwei Jahren, nachdem der Anspruch auf die Altersrente aus dem Rentenversicherungssystem entstand, berechnet werden. Ab diesem Zeitpunkt kann der Versicherte einen Ausdienzuschuß in der Höhe des Unterschiedes zwischen der Altersrente, welche ihm zusteht, und der Höhe des Ausdienzuschusses, die zu diesem Zeitpunkt beansprucht werden kann, erhalten. Ab diesem Datum erhöht sich auch die Ausdienzulage separat und unabhängig von der Rentenhöhe. Bei der Berechnung der Höhe der Ausdienzulage, die neben der 
Altersrente aus der Rentenversicherung ausgezahlt werden soll, wird innerhalb der ersten zwei Jahre, nachdem der Versicherte Altersrente beanspruchen kann, als Referenzhöhe die volle Höhe der Ausdienzulage zugrunde gelegt.

\section{Bewertung}

Das allgemeine Rentenversicherungssystem, dessen Versichertenkreis alle Erwerbstätigen ohne Unterschied hinsichtlich der Art der Erwerbstätigkeit erfaßt, gewährleistet die gleiche Absicherung bei Invalidität, im Alter sowie im Fall des Todes des Versicherten und somit eine Gleichstellung der Bürger der Tschechischen Republik im Hinblick auf die soziale Absicherung bei Eintritt der genannten Risiken. Die Tschechische Republik mißt dem Gleichbehandlungsgrundsatz eine große Bedeutung bei, weswegen die Errichtung spezieller Versicherungssysteme für bestimmte Gruppen von Erwerbstätigen vom politischen Blickwinkel aus betrachtet nicht durchsetzbar ist. Diesbezüglich sind daher in der nächsten Zeit keine größeren Änderungen oder Reformen der Beamtenversorgung zu erwarten.

Zwar kennt das tschechische Recht ein Berufsbeamtentum, der Theorie nach könnten daher gleiche Folgerungen hinsichtlich der Altersabsicherung der Beamten gezogen werden wie für Deutschland. Doch haben Beamte keine besondere, verfassungsrechtlich begründete Stellung, aus der sich eine Verpflichtung des Staates zur Errichtung besonderer Alterssicherungssysteme herleiten ließe. Die Verfassung der Tschechischen Republik schreibt in ihrem Art. 79 Abs. 2 lediglich vor, daß die Rechtsverhältnisse der Staatsangestellten in Ministerien sowie in anderen Verwaltungsbehörden durch Gesetz geregelt werden sollen. Zudem ist die tschechische Gesellschaft auf Gewährleistung einheitlichen Rechts für jeden Bürger bedacht. Ferner durchlebt die Finanzierung des öffentlichen Haushalts derzeit eine Krise. Durch die gegenwärtige wirksame Umsetzung der Rentenversicherung haben deshalb ehemalige Beamte, die mittlerweile im Ruhestand sind, keine besondere Stellung. Die sog. Ausdienzulage für Staatsbedienstete ist nicht Teil der allgemeinen Rentenversicherung.

\section{Weiterführende Literatur}

Kocourek, J.: Služební zákon - o službě státních zaměstnanců ve správních úřadech a jejich odměňování (Dienstgesetz), Eurounion 2005

Samek, V; Korotvičková, M.; Kubínková M.; Onisko J.: Zákon o úřednících územních samosprávných celků (Gesetz über Beamte in territorialen Selbstverwaltungseinheiten), Sondy 2002

Trykar, L.: Služební poměr státních zaměstnanců (Dienstgesetz), Leges 2008 


\title{
Die Versorgung der Civil Servants im Vereinigten Königreich
}

\author{
Martin Landauer
}

I. Grundlegung

1. Civil Service als Vergleichsgruppe

2. Rechtliche Stellung der Civil Servants

3. Alterssicherung der Civil Servants im Kontext allgemeiner sozialer Sicherung

II. Ausgestaltung der Alterssicherung der Civil Servants

1. State pensions
a) Basic State Pension
b) State Second Pension
c) Umwandlung von Renten in Zahlung einer Pauschalsumme

2. Betriebliche Altersversorgung
a) Allgemeines
b) Das Principle Civil Service Pension Scheme

3. Private Rentenkonten

a) Personal Pensions und Stakeholder Pensions

b) Partnership pension accounts

c) Civil Service Additional Voluntary Contribution Scheme

III. Bewertung 